\title{
Epidemiological Characterization of a Fourth Wave of Pandemic A/H1N1 Influenza in Mexico, Winter 2011-2012: Age Shift and Severity
}

\author{
Víctor H. Borja-Aburto ${ }^{a}$, Gerardo Chowell ${ }^{b, c}$, Cécile Viboud ${ }^{b}$, Lone Simonsen ${ }^{b, d}$, Mark A. \\ Miller $^{b}$, Concepción Grajales-Muñiz ${ }^{a}$, Cesar R. González-Bonilla ${ }^{a}$, Jose A Diaz-Quiñonez ${ }^{a}$, \\ and Santiago Echevarría-Zuno ${ }^{e}$
}

aCoordinación de Vigilancia Epidemiológica y Apoyo en Contingencias, Instituto Mexicano del Seguro Social, Mexico, D.F., Mexico bivision of Epidemiology and Population Studies, Fogarty International Center, National Institutes of Health, Bethesda, Maryland ${ }^{\mathrm{C}}$ Mathematical, Computat ional and Modeling Sciences Center, School of Human Evolution and Social Change, Arizona State University, Tempe, Arizona dDepartment of Global Health, School of Public Health and Health Services, George Washington University, Washington, D.C e Dirección de Prestaciones Médicas, Instituto Mexicano del Seguro Social, México, D.F., México

\begin{abstract}
Background and Aims-A substantial recrudescent wave of pandemic influenza A/H1N1 affected the Mexican population from December 1, 2011-March 20, 2012 following a 2-year period of sporadic transmission.

Methods-We analyzed demographic and geographic data on all hospitalizations with severe acute respiratory infection (SARI) and laboratory-confirmed A/H1N1 influenza, and inpatient deaths, from a large prospective surveillance system maintained by a Mexican social security medical system during April 1, 2009- March 20, 2012. We also estimated the reproduction number $(\mathrm{R})$ based on the growth rate of the daily case incidence by date of symptoms onset.
\end{abstract}

\begin{abstract}
Results-A total of 7569 SARI hospitalizations and 443 in-patient deaths (5.9\%) were reported between December 1, 2011, and March 20, 2012 (1115 A/H1N1-positive inpatients and 154 A/ H1N1-positive deaths). The proportion of laboratory-confirmed A/H1N1 hospitalizations and deaths was higher among subjects $\Varangle 60$ years of age $\left(\chi^{2}\right.$ test, $\left.p<0.0001\right)$ and lower among younger age groups ( $\chi^{2}$ test, $p<0.04$ ) for the 2011-2012 pandemic wave compared to the earlier waves in 2009. The reproduction number of the winter 2011-2012 wave in central Mexico was estimated at 1.2-1.3, similar to that reported for the fall 2009 wave, but lower than that of spring 2009.
\end{abstract}

Conclusions-We documented a substantial increase in the number of SARI hospitalizations during the period December 2011-March 2012 and an older age distribution of laboratoryconfirmed A/H1N1 influenza hospitalizations and deaths relative to $2009 \mathrm{~A} / \mathrm{H} 1 \mathrm{~N} 1$ pandemic

(C) 2012 Instituto Mexicano del Seguro Social (IMSS). Published by Elsevier Inc. All rights reserved.

Address reprint requests to: Gerardo Chowell, Associate Professor, Arizona State University, University Ave., Tempe, AZ; Phone: NEEDED; FAX: NEEDED; gchowell@asu.edu.

Competing interests. The authors declare no competing interests relevant to this article.

Publisher's Disclaimer: This is a PDF file of an unedited manuscript that has been accepted for publication. As a service to our customers we are providing this early version of the manuscript. The manuscript will undergo copyediting, typesetting, and review of the resulting proof before it is published in its final citable form. Please note that during the production process errors may be discovered which could affect the content, and all legal disclaimers that apply to the journal pertain. 
patterns. The gradual change in the age distribution of $\mathrm{A} / \mathrm{H} 1 \mathrm{~N} 1$ infections in the post-pandemic period is consistent with a build-up of immunity among younger populations.

\section{Keywords}

A/H1N1 influenza pandemic; Hospitalizations; Deaths; Age distribution; Transmissibility

\section{Introduction}

The swine-origin pandemic A/H1N1 influenza virus caused a sizable epidemic in Mexico in winter 2011-2012 following a 2-year period of sporadic transmission. Mexico experienced a series of three A/H1N1 pandemic waves in the spring, summer, and fall of 2009 (1-3) followed by a large pandemic vaccination campaign towards the end of 2009. These three waves were together associated with a high excess mortality burden relative to that seen in other countries (4-6). Because a significant fraction of the population is now protected from $\mathrm{A} / \mathrm{H} 1 \mathrm{~N} 1$ influenza through natural exposure or vaccination (7), there is potential for the emergence of drift A/H1N1 influenza variants and/or changing age patterns as typically seen during post-pandemic periods $(8,9)$.

Here we report on the epidemiology of a recrudescent (fourth) wave of pandemic A/H1N1 influenza activity in Mexico from December 1, 2011-March 20, 2012 and update our preliminary findings based on data up to February 10, 2012 (10). Because past pandemic experiences have indicated substantial post-pandemic morbidity and mortality burden may occur months to years after the initial pandemic waves $(9,11-14)$, we must remain vigilant and continue to monitor the virological characteristics of the $2009 \mathrm{~A} / \mathrm{H} 1 \mathrm{~N} 1$ influenza virus (10,15-17) and compare the epidemiological characteristics of laboratory-confirmed A/ H1N1 hospitalizations and deaths in winter 2011-2012 with those previously reported for the 2009 pandemic waves and show a significant change in the age distribution of cases and deaths.

\section{Materials and Methods}

\section{Epidemiological Data}

Individual level hospitalization data were available from a prospective epidemiological surveillance system that was put into place especially for the 2009 influenza pandemic by the Mexican Institute for Social Security (IMSS) $(4,18,19)$. IMSS is a tripartite Mexican health system covering approximately $40 \%$ of the Mexican population comprising workers in the private sector and their families, relying on a network of 1,099 primary health-care units and 259 hospitals nationwide. The age and gender distributions of persons affiliated with the IMSS medical system are representative of the general Mexican population (4).

We analyzed information from all hospitalizations and in-patient deaths among patients admitted with severe acute respiratory infection (SARI) during December 1, 2011-March 20,2012 . SARI was defined in a person with respiratory difficulty presenting fever $38^{\circ} \mathrm{C}$ and cough together with one or more of the following clinical symptoms: confinement to bed, thoracic pain, polypnea, or acute respiratory distress syndrome. Children $<5$ years of age with pneumonia or severe pneumonia that required hospitalization were also considered as SARI cases. Respiratory swabs were obtained for about $26 \%$ of SARI hospitalizations in winter 2011-2012 and were tested for the influenza virus by rRT-PCR (20). For all SARI hospitalizations, we retrieved demographic information (age in years and gender), influenza laboratory test result (if tested), reporting state (including 31 states plus the Federal District), and dates of symptom onset (self-reported). We also obtained population data by state and age group for all persons affiliated with the IMSS in 2009 to calculate incidence rates. 


\section{Age Distribution and Severity of A/H1N1 Influenza in 2009 and 2011-2012}

We examined the age distribution of hospitalizations and deaths based on all cases of SARI and laboratory-confirmed A/H1N1 influenza patients reported from December 1, 2011March 20, 2012. We compared the age distribution of hospitalizations and deaths in winter 2011-2012 with those described for the three waves of the 2009-2010 A/H1N1 pandemic in Mexico 8 (April 1, 2009-March 31, 2010) using the same IMSS reporting system. We also calculated the in-hospital case fatality rate by dividing inpatient deaths by hospitalizations, separately for SARI and laboratory-confirmed A/H1N1.

\section{Spatial Distribution of A/H1N1 Influenza in Winter 2011-2012 and Reproduction Number Estimate}

We analyzed state- and age-specific time series of laboratory-confirmed A/H1N1 influenza hospitalizations according to day of symptom onset to analyze the geographic dissemination patterns of the fourth pandemic wave of A/H1N1 influenza transmission in Mexico from December 1, 2011-March 20, 2012. Further, we estimated the reproduction number ${ }^{\circledR}$ in central Mexico where the great majority of cases have been reported, based on a simple method previously used in the context of the 2009 A/H1N1 pandemic waves in Mexico (4).

Specifically, we estimate the initial epidemic growth rate by fitting an exponential function to the early ascending phase of daily SARI or A/H1N1 hospitalizations by date of symptom onset (21). The early ascending phase was determined as the period between the day of pandemic onset and the midpoint between the onset and peak days. We assumed a mean generation interval of 3 and 4 days, which are within the range of mean estimates for the 2009 influenza pandemic $(2,22-24)$. As a sensitivity analysis we also assessed small variations in the length of the ascending epidemic phase used to estimate the exponential growth rate ( \pm 4 days). Statistical analyses were performed using SPSS v.20.0 and Matlab (The Mathworks, Inc.).

\section{Results}

\section{Overall Epidemiological Patterns}

The characteristics of all SARI and A/H1N1-positive hospitalizations reported to the IMSS medical system between December 1, 2011 and March 20, 2012 are presented in Table 1. The time series of daily SARI hospitalizations and deaths and laboratory-confirmed influenza hospitalizations are shown in Figures 1 and 2, respectively. An A/H1N1 influenza outbreak began around December 1, 2011 and lasted for 15 weeks (Figure 3), affecting primarily central Mexico (Figure 1). The daily number of SARI hospitalizations in winter 2011-2012 exceeded the peak of the major fall wave of the 2009 A/H1N1 influenza pandemic but declined at a faster rate (Supplementary Figure 1). In Mexico City the cumulative number of SARI hospitalizations during December 1, 2011-March 20, 2012 represents $44.5 \%$ of all SARI hospitalizations that were reported in Mexico City during the first year of A/H1N1 virus circulation (April 2009-March 2010).

\section{Severity of Disease}

A total of 7,569 SARI hospitalizations and 443 in-patient SARI deaths (case fatality rate, 5.9\% [95\% CI: 5.3, 6.4]) were reported to the IMSS system between December 1, 2011 and March 20, 2012. Our case fatality rate for laboratory-confirmed A/H1N1 inpatients was $13.8 \%$ (95\% CI: 11.7, 15.9) during the same period (1115 in-patients and 154 deaths). Our estimate of the case fatality rate (CFR) for hospitalized A/H1N1 patients in 2011-2012 is not significantly different from the CFR measured in 2009 (16.1\% [95\% CI: 15.0, 17.2]). 


\section{Age}

Overall, the majority of laboratory-confirmed influenza inpatients during December 1, 2011-March 20, 2012 were among persons aged 15-59 years (64.4\%) followed by the 0 - to 4-year-old age group (13.4\%) and persons $\succeq 60$ years $(13.7 \%)$ (Table 1$)$. Severity increased with older age, with an inpatient fatality rate of $22.3 \%$ (95\% CI: 16.5, 28.0) for persons $\ 60$ years.

The cumulative hospitalization and inpatient death rates for the three waves of the 2009$2010 \mathrm{~A} / \mathrm{H} 1 \mathrm{~N} 1$ pandemic are on average 4 and 4.6 times greater than the corresponding rates for the 2011-2012 A/H1N1 wave in Mexico. Comparison of the age-specific A/H1N1 hospitalization and death rates reveals an increasing burden among older populations in 2011-2012, relative to the 2009-2010 waves (Supplementary Figure 2). An analysis of the proportionate distribution of $\mathrm{A} / \mathrm{H} 1 \mathrm{~N} 1$ hospitalization and inpatient deaths reveals a shift in the age distribution of recent cases towards older ages as well. Specifically, we note a significantly higher proportion of individuals $>60$ years of age hospitalized with laboratoryconfirmed A/H1N1 in 2011-2012, relative to the 2009-2010 pandemic period (13.7 vs. $6.1 \%, \chi^{2}$ test, $p<0.0001$, Table 2, Figure 4), and a slight but significant increase in A/H1N1 hospitalizations among infants $0-4$ years of age compared to the 2009 pandemic (13.4 vs. $10.1 \%, \chi^{2}$ test, $p=0.002$ ). We also found a reduction in the proportion of $\mathrm{A} / \mathrm{H} 1 \mathrm{~N} 1$-positive hospitalizations among persons 5-14 years of age compared to the 2009 pandemic $(8.5 \%$ vs. $14.9 \%, \chi^{2}$ test, $\left.p=0.0003\right)$. We found similar changes in the age distribution of $\mathrm{A} / \mathrm{H} 1 \mathrm{~N} 1$ inpatient deaths in 2011-2012 compared to the 2009 A/H1N1 influenza pandemic (Supplementary Table 1, Figure 4). Specifically, 27.3\% of deaths occurred among persons $\checkmark 60$ years of age in the winter 2011-2012 epidemic period, whereas only $8.9 \%$ occurred in the 2009-2010 period ( $\chi^{2}$ test, $p<0.0001$ ). Similar to the age shift in hospitalization data, the proportion of $\mathrm{A} / \mathrm{H} 1 \mathrm{~N} 1$ inpatient deaths among individual aged 15-44 years declined, relative to $2009-2010$ (31.8 vs. $52.9 \%, \chi^{2}$ test, $\left.p<0.01\right)$.

\section{Geography}

The majority of A/H1N1 inpatients during the fourth wave were reported in central Mexican states $(55.1 \%)$ followed by southeastern states $(27.9 \%)$ (Table 1$)$ and a higher proportion of $\mathrm{A} / \mathrm{H} 1 \mathrm{~N} 1$ deaths occurred in central states compared to other regions $\left(\chi^{2}\right.$ test, $p=0.04$ ).

\section{Estimates of the Reproduction Number}

Assuming a mean generation interval of 3 (and 4) days, the mean $\mathrm{R}$ was estimated to be 1.2 (1.3) during the period from December 17, 2011-January 9, 2012, based on daily A/H1N1positive hospitalizations. As a sensitivity analysis we also estimated $\mathrm{R}$ using daily SARI hospitalizations; our estimate of $\mathrm{R}$ was somewhat lower at 1.1 (1.2). When the length of the epidemic ascending phase was varied ( \pm 4 days), our $R$ estimates changed by 0.1 or less.

\section{Discussion}

We characterized the epidemiology of a recrudescent fourth wave of A/H1N1 influenza transmission in Mexico spanning December 1, 2011-March 20, 2012, based on hospitalizations for acute respiratory infections and laboratory-confirmed A/H1N1 infections. We compared the impact, severity, age patterns, and reproduction number of this fourth wave with those of earlier pandemic waves in spring, summer and fall 2009 in Mexico $(1,4)$. We used individual-level patient information collected through a prospective influenza surveillance system put into place especially for the 2009 pandemic by the largest Mexican Social Security medical system and providing daily data during the period from 2009- 2012 (18). Our data show that the nationwide peak level of daily SARI hospitalizations in 2012 exceeded the peak of SARI hospitalizations observed during the 
major fall pandemic wave in 2009. We also documented a significant increase in the proportion of $\mathrm{A} / \mathrm{H} 1 \mathrm{~N} 1$ hospitalizations and deaths among persons $\checkmark 60$ years of age relative to the 2009 pandemic, and a significant reduction in the proportion of $\mathrm{A} / \mathrm{H} 1 \mathrm{~N} 1$ hospitalizations and deaths among younger age groups. The age shift seen in the 2011-2012 winter season most likely reflects a build-up of immunity among younger populations, a pattern similar to that reported among A/H1N1 hospitalized patients during the first postpandemic season in Spain (17). Yet, this epidemiological pattern may also be indicative of a gradual emergence of drift $\mathrm{A} / \mathrm{H} 1 \mathrm{~N} 1$ variants. A recent study found that minor changes in the hemagglutinin of $2009 \mathrm{~A} / \mathrm{H} 1 \mathrm{~N} 1$ influenza virus are sufficient to alter its antigenic properties (15). Whether the observed age shift is due to a build-up of immunity or changes in the A/ H1N1 influenza virus composition has implications for influenza prevention and mitigation strategies, which we discuss below.

The observed change in age distribution of hospitalization and deaths in the post-pandemic period of 2009 is reminiscent of the influenza seasons following the 1918 influenza pandemic $(8,9,25)$ and the 1968 pandemic (26), which highlights the importance of closely monitoring epidemiological and virological changes in the A/H1N1 influenza virus. A quantitative analysis of excess mortality prior to and after the1918 influenza pandemic found that the age distribution of influenza-related mortality returned to pre-pandemic mortality levels a few years after the initial pandemic waves as a result of emerging drift variants $(9,27)$.

During the first year of circulation of the 2009 A/H1N1 influenza pandemic virus, protection from influenza-related morbidity and mortality rates was reported in persons $>60$ years of age. This phenomenon of senior sparing in age cohorts born prior to the 1957 pandemic is consistent with first exposure to antigenically related $\mathrm{A} / \mathrm{H} 1 \mathrm{~N} 1$ viruses in childhood, a pattern consistent with the antigen recycling and original antigenic sin hypotheses $(1,28-31)$. A high fraction of the Mexican population is now protected against the 2009 A/H1N1 influenza virus through natural exposure in 2009 (children and young adults) or prior immunity (seniors) (7) and by pandemic vaccines. Over 8 million doses of seasonal influenza vaccine (featuring a good match for the H1N1-pdm vaccine component) were administered in 20112012 winter (35\% vaccination coverage among IMSS-affiliated seniors 260 years of age, $70 \%$ among those $<5$ years of age, $40 \%$ among those $50-59$ years of age, and $24 \%$ among those 5-9 years of age).

Although we saw evidence of a shift in the age distribution of 2011-2012 cases towards seniors, the absolute risk of being hospitalized was still relatively low in this age group, relative to those in younger adults. The declining rates of severe cases in younger age groups is most consistent with a build-up of immunity.

Overall, the age distribution of the fourth wave of $\mathrm{A} / \mathrm{H} 1 \mathrm{~N} 1$ influenza hospitalizations and deaths in Mexico is relatively flat and not quite back to the normal J-shaped age risk profile that characterizes seasonal influenza. In the long run, we expect the pandemic A/H1N1 virus to drift genetically to escape mounting population immunity - perhaps with the result that seniors are no longer protected (1). Hence, the epidemiological evidence is consistent with the genetic and antigenic information published on circulating influenza virus, suggesting a lack of antigenic drift in A/H1N1 viruses in Mexico or elsewhere in winter 2011-2012, a season associated with relatively low A/H1N1 activity globally (32).

Because transmission of the A/H1N1 influenza virus was sporadic in the winter of 20102011 in Mexico, we cannot rule out the possibility of some loss of population immunity since 2009. We estimated a reproduction number for the fourth $\mathrm{A} / \mathrm{H} 1 \mathrm{~N} 1$ wave to be significantly lower to that of the spring $(\mathrm{R} \sim 1.8-2.1)$ and summer $(\mathrm{R} \sim 1.6-1.9)$ pandemic 
waves in 2009 in Mexico, but in close agreement with estimates of the fall (third) 2009 wave $(\mathrm{R} \sim 1.2-1.3)$ (4).

Perhaps the most surprising finding of this analysis is the occurrence of a substantial fourth wave of pandemic A/H1N1 activity in Mexico, a country that has already experienced severe excess mortality impact during three waves of transmission in $2009(4,6)$. Although we are just beginning to assess the global mortality burden of the $2009 \mathrm{~A} / \mathrm{H} 1 \mathrm{~N} 1$ virus in the pandemic and post-pandemic period, important geographical variations in the number, timing, transmissibility and impact of sequential pandemic waves are obvious. For instance, the UK experienced two waves in spring and fall 2009 , to be followed by a relatively severe recrudescent wave in 2010-2011 not seen in other European countries (33). The U.S. experienced the brunt of the pandemic burden in the first year of A/H1N1 circulation. To our knowledge, the four-wave pattern seen in Mexico in 2009-2012 has not been reported in other countries. Whether these differences can be explained by geographical variations in prior immunity, seasonal drivers, control strategies, connectivity, health and healthcare is unclear and remains a key area for future research.

In summary, our findings indicate a changing age distribution of laboratory-confirmed A/ H1N1 influenza hospitalizations and deaths in winter 2011-2012, relative to 2009-2010 A/ H1N1 pandemic patterns. The proportion of hospitalizations and deaths is increasing in persons $\geq 60$ years of age, an age group that was largely protected during the early pandemic waves in 2009. In contrast, rates of A/H1N1 hospitalizations and deaths are declining among younger population groups, consistent with a gradual build-up of immunity. This gradual change in the age distribution of A/H1N1 influenza in 2011-2012 in Mexico is reminiscent of post-pandemic patterns in past influenza pandemics. Nevertheless, the severity of the fourth wave was not different from the previous waves in terms of mortality. By contrast, during the 1889 pandemic, the third wave occurring in the winter of 1991-1992 was far more deadly than previous waves $(14,34)$.

The dominant pattern of A/H1N1 influenza activity in winter 2011-2012 in Mexico is unique. By contrast, A/H3N2 influenza has driven winter epidemics in the U.S., Canada (together with influenza B viruses), Europe, North Africa and the Eastern Mediterranean Region, whereas influenza B transmission has predominated in China (35). A multinational comparison of the epidemiology of pandemic and post-pandemic waves would be useful to shed light on the long-term transmission dynamics and build-up of immunity to pandemic viruses and report control strategies.

\section{Supplementary Material}

Refer to Web version on PubMed Central for supplementary material.

\section{Acknowledgments}

This research was conducted in the context of the MISMS (Multinational Influenza Seasonal Mortality Study), an ongoing international collaborative effort to understand influenza epidemiological and evolutionary patterns, led by the Fogarty International Center, National Institutes of Health (http://www.origem.info/misms/index.php). The MISMS study is funded by the International Influenza Unit, Office of Global Health Affairs, Department of Health and Human Services. LS acknowledges support from the RAPIDD (Research and Policy for Infectious Disease Dynamics) program of the Science and Technology Directorate, Department of Homeland Security, and the Fogarty International Center.

\section{References}

1. Chowell G, Bertozzi SM, Colchero MA, et al. Severe respiratory disease concurrent with the circulation of H1N1 influenza. N Engl J Med. 2009; 361:674-679. [PubMed: 19564633] 
2. Fraser C, Donnelly CA, Cauchemez S, et al. Pandemic potential of a strain of influenza A (H1N1): early findings. Science. 2009; 324:1557-1561. [PubMed: 19433588]

3. Perez-Padilla R, de la Rosa-Zamboni D, Ponce de Leon S, et al. Pneumonia and respiratory failure from swine-origin influenza A (H1N1) in Mexico. N Engl J Med. 2009; 361:680-689. [PubMed: 19564631]

4. Chowell G, Echevarría-Zuno S, Viboud C, et al. Characterizing the epidemiology of the 2009 influenza A/H1N1 pandemic in Mexico. PloS Medicine. 2011; 8:e1000436. [PubMed: 21629683]

5. Zepeda-Lopez HM, Perea-Araujo L, Miliar-Garcia A, et al. Inside the outbreak of the 2009 influenza A (H1N1)v virus in Mexico. PLoS One. 2010; 5:e13256. [PubMed: 20949040]

6. Charu V, Chowell G, Palacio Mejia LS, et al. Mortality burden of the A/H1N1 pandemic in Mexico: a comparison of deaths and years of life lost to seasonal influenza. Clin Infect Dis. 2011; 53:985993. [PubMed: 21976464]

7. Elizondo-Montemayor L, Alvarez MM, Hernandez-Torre M, et al. Seroprevalence of antibodies to influenza A/H1N1/2009 among transmission risk groups after the second wave in Mexico, by a virus-free ELISA method. Int J Infect Dis. 2011; 15:e781-e786. [PubMed: 21855383]

8. Simonsen L, Clarke MJ, Schonberger LB, et al. Pandemic versus epidemic influenza mortality: a pattern of changing age distribution. J Infect Dis. 1998; 178:53-60. [PubMed: 9652423]

9. Saglanmak N, Andreasen V, Simonsen L, et al. Gradual changes in the age distribution of excess deaths in the years following the 1918 influenza pandemic in Copenhagen: using epidemiological evidence to detect antigenic drift. Vaccine. 2011; 29(suppl 2):B42-B48. [PubMed: 21757103]

10. Chowell G, Echevarria-Zuno S, Viboud C, et al. Recrudescent wave of pandemic A/H1N1 influenza in Mexico, winter 201-2012: age shift and severity. PLoS Curr. 2012; 4:RRN1306. [PubMed: 22485199]

11. Olson DR, Simonsen L, Edelson PJ, et al. Epidemiological evidence of an early wave of the 1918 influenza pandemic in New York City. Proc Natl Acad Sci USA. 2005; 102:11059-11063. [PubMed: 16046546]

12. Chowell G, Viboud C, Simonsen L, et al. Mortality patterns associated with the 1918 influenza pandemic in Mexico: evidence for a spring herald wave and lack of preexisting immunity in older populations. J Infect Dis. 2010; 202:567-575. [PubMed: 20594109]

13. Chowell G, Viboud C, Simonsen L, et al. The 1918-1920 influenza pandemic in Peru. Vaccine. 2011; 29(suppl 2):B21-B26. [PubMed: 21757099]

14. Valleron AJ, Cori A, Valtat S, et al. Transmissibility and geographic spread of the 1889 influenza pandemic. Proc Natl Acad Sci USA. 2010; 107:8778-8781. [PubMed: 20421481]

15. Strengell M, Ikonen N, Ziegler T, et al. Minor changes in the hemagglutinin of influenza A(H1N1)2009 virus alter its antigenic properties. PLoS One. 2011; 6:e25848. [PubMed: 22022458]

16. Nelson M, Spiro D, Wentworth D, et al. The early diversification of influenza A/H1N1pdm. PLoS Curr. 2009; 1:RRN1126. [PubMed: 20029664]

17. Viasus D, Cordero E, Rodriguez-Bano J, et al. Changes in epidemiology, clinical features and severity of influenza A (H1N1) 2009 pneumonia in the first post-pandemic influenza season. Clin Microbiol Infect. 2012; 18:E55-E62. [PubMed: 22264321]

18. Echevarria-Zuno S, Mejia-Arangure JM, Mar-Obeso AJ, et al. Infection and death from influenza A H1N1 virus in Mexico: a retrospective analysis. Lancet. 2009; 374:2072-2079. [PubMed: 19913290]

19. Chowell G, Viboud C, Simonsen L, et al. Impact of antiviral treatment and hospital admission delay on the risk of death of 2009 A/H1N1 pandemic influenza in Mexico, April-December 2009. BMC Infect Dis. 2011; 12:97. [PubMed: 22520743]

20. Centers for Disease Control and Prevention. Serum cross-reactive antibody response to a novel influenza A (H1N1) virus after vaccination with seasonal influenza vaccine. MMWR Morb Mortal Wkly Rep. 2009; 58:521-524. [PubMed: 19478718]

21. Wallinga J, Lipsitch M. How generation intervals shape the relationship between growth rates and reproductive numbers. Proc Biol Sci. 2007; 274:599-604. [PubMed: 17476782]

22. Yang Y, Sugimoto JD, Halloran ME, et al. The transmissibility and control of pandemic influenza A (H1N1) virus. Science. 2009; 326:729-733. [PubMed: 19745114] 
23. Cauchemez S, Donnelly CA, Reed C, et al. Household transmission of 2009 pandemic influenza A (H1N1) virus in the United States. N Engl J Med. 2009; 361:2619-2627. [PubMed: 20042753]

24. Cowling BJ, Chan KH, Fang VJ, et al. Comparative epidemiology of pandemic and seasonal influenza A in households. N Engl J Med. 2010; 362:2175-2184. [PubMed: 20558368]

25. Simonsen L, Reichert TA, Miller M. The virtues of antigenic sin: consequences of pandemic recycling on influenza-associated mortality: options for the control of influenza V: International Congress Series. 2004; 1263:791-794.

26. Viboud C, Grais RF, Lafont BA, et al. Multinational impact of the 1968 Hong Kong influenza pandemic: evidence for a smoldering pandemic. J Infect Dis. 2005; 192:233-248. [PubMed: 15962218]

27. Andreasen V, Viboud C, Simonsen L. Epidemiologic characterization of the 1918 influenza pandemic summer wave in Copenhagen: implications for pandemic control strategies. J Infect Dis. 2008; 197:270-278. [PubMed: 18194088]

28. Vaillant L, La Ruche G, Tarantola A, et al. Epidemiology of fatal cases associated with pandemic H1N1 influenza 2009. Euro Surveill. 2009; 14(33)

29. Viboud C, Miller M, Olson D, et al. Preliminary estimates of mortality and years of life lost associated with the $2009 \mathrm{~A} / \mathrm{H} 1 \mathrm{~N} 1$ pandemic in the US and comparison with past influenza seasons. PLoS Curr. 2010:RRN1153.

30. Miller MA, Viboud C, Balinska M, et al. The signature features of influenza pandemics-implications for policy. N Engl J Med. 2009; 360:2595-2598. [PubMed: 19423872]

31. Reichert T, Chowell G, Nishiura H, et al. Does glycosylation as a modifier of original antigenic sin explain the case age distribution and unusual toxicity in pandemic novel H1N1 influenza? BMC Infect Dis. 2010; 10:5. [PubMed: 20059763]

32. Centers for Disease Control and Prevention. FluView. 2011-2012 Influenza season Week 5 ending February 4, 2012. Website: http://www.cdc.gov/flu/weekly/. [cited; Available from: http:// www.cdc.gov/flu/weekly/

33. Ellis J, Galiano M, Pebody R, et al. Virological analysis of fatal influenza cases in the United Kingdom during the early wave of influenza in winter 2010/11. Euro Surveill. 2011; 16(1)

34. Stuart-Harris CH. Pandemic influenza: an unresolved problem in prevention. J Infect Dis. 1970; 122:108-115. [PubMed: 4914941]

35. Centers for Disease Control and Prevention. Seasonal Flu: International Situation Update, 20112012 Influenza season. Website: http://www.cdc.gov/flu/international/activity.htm. [cited; Available from: http://www.cdc.gov/flu/weekly/ 

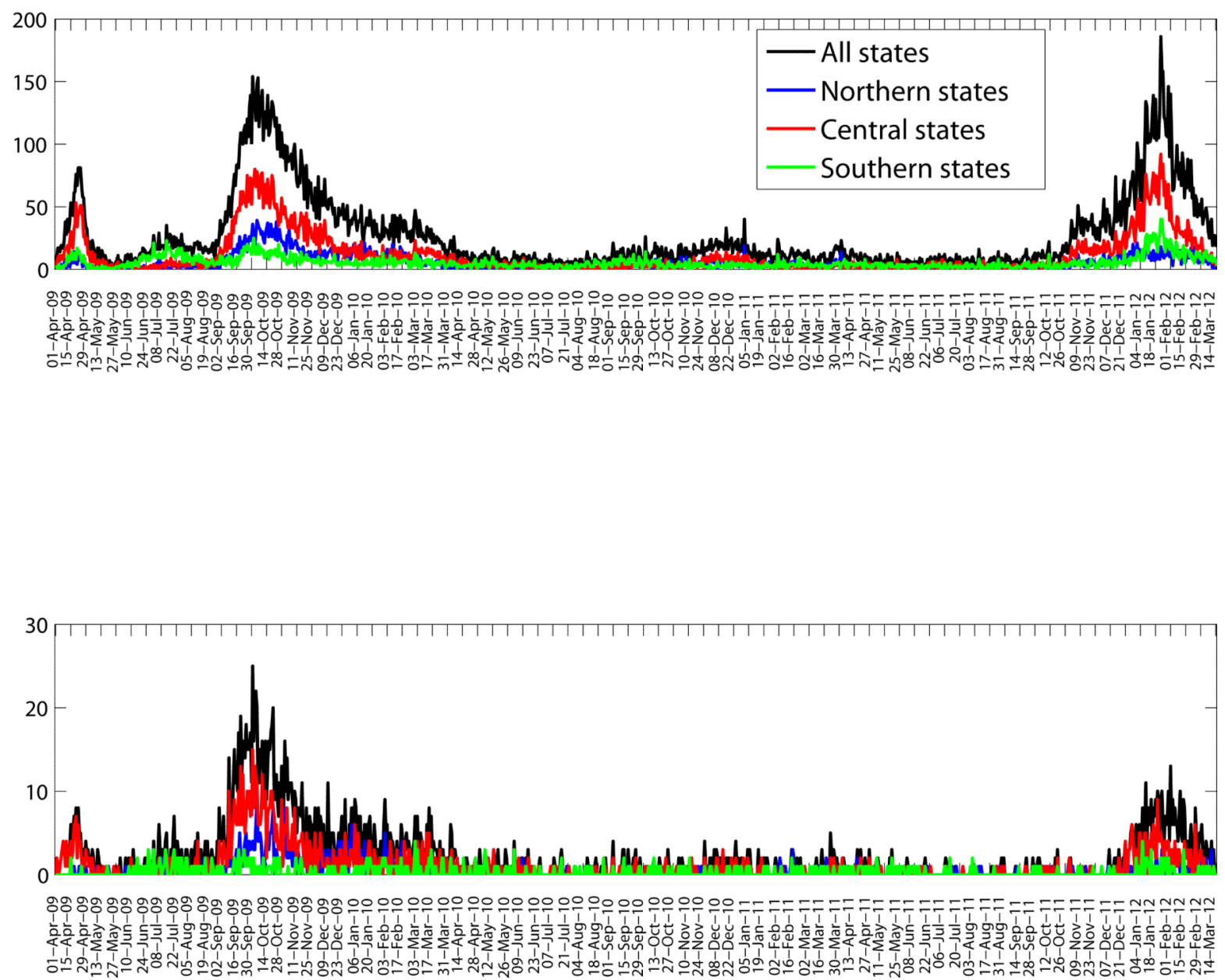

Figure 1.

Daily epidemic curves of all SARI hospitalizations (top) and deaths (bottom) according to dates of symptom onset in northern, central, and southeastern states of Mexico (April 1, 2009-March 20, 2012). 


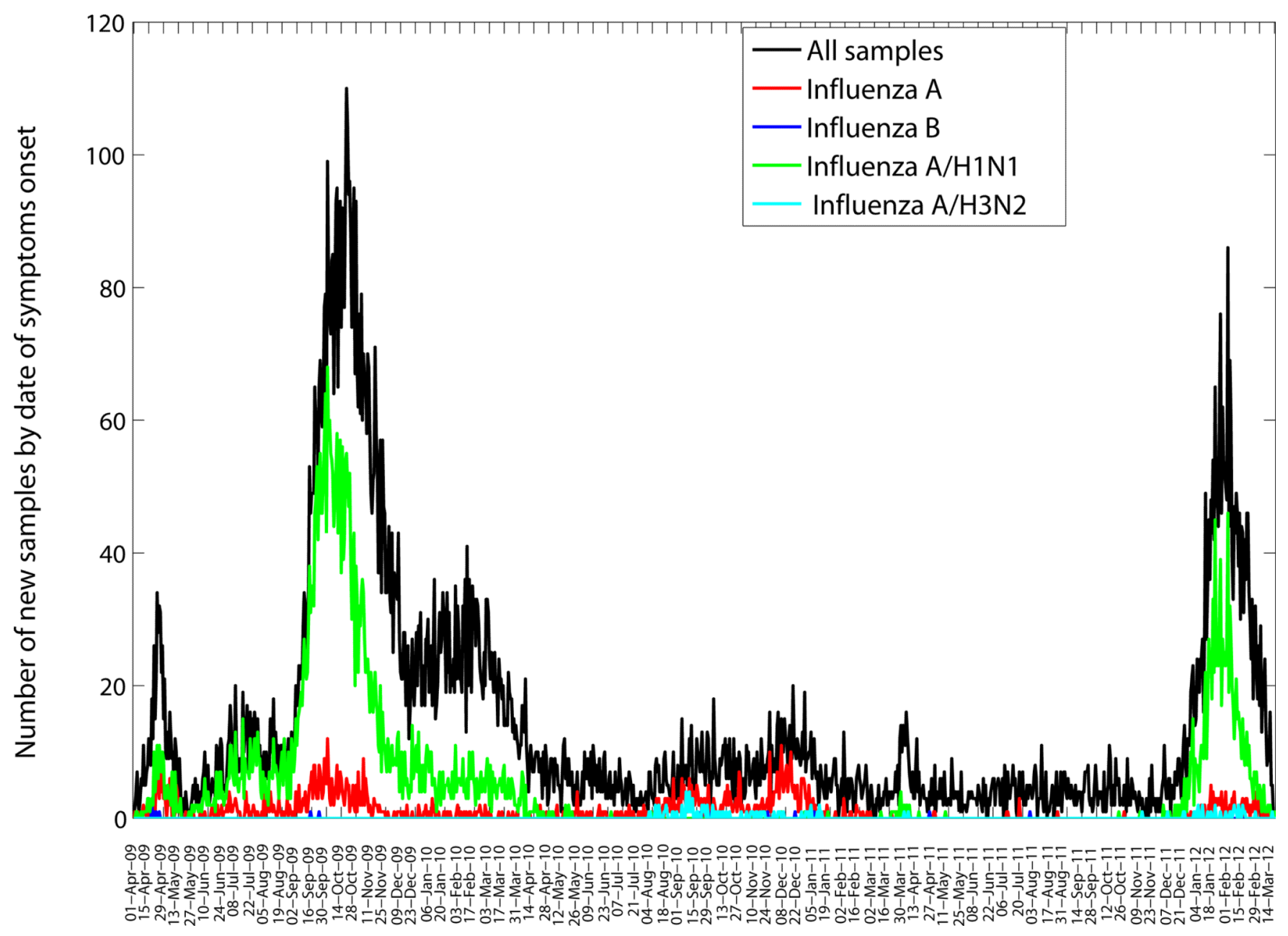

Figure 2.

Daily number of influenza tests among SARI hospitalizations and laboratory-confirmed influenza hospitalizations according to dates of symptom onset (April 1, 2009-March 20, 2012) in the 32 Mexican states according to influenza subtype. 


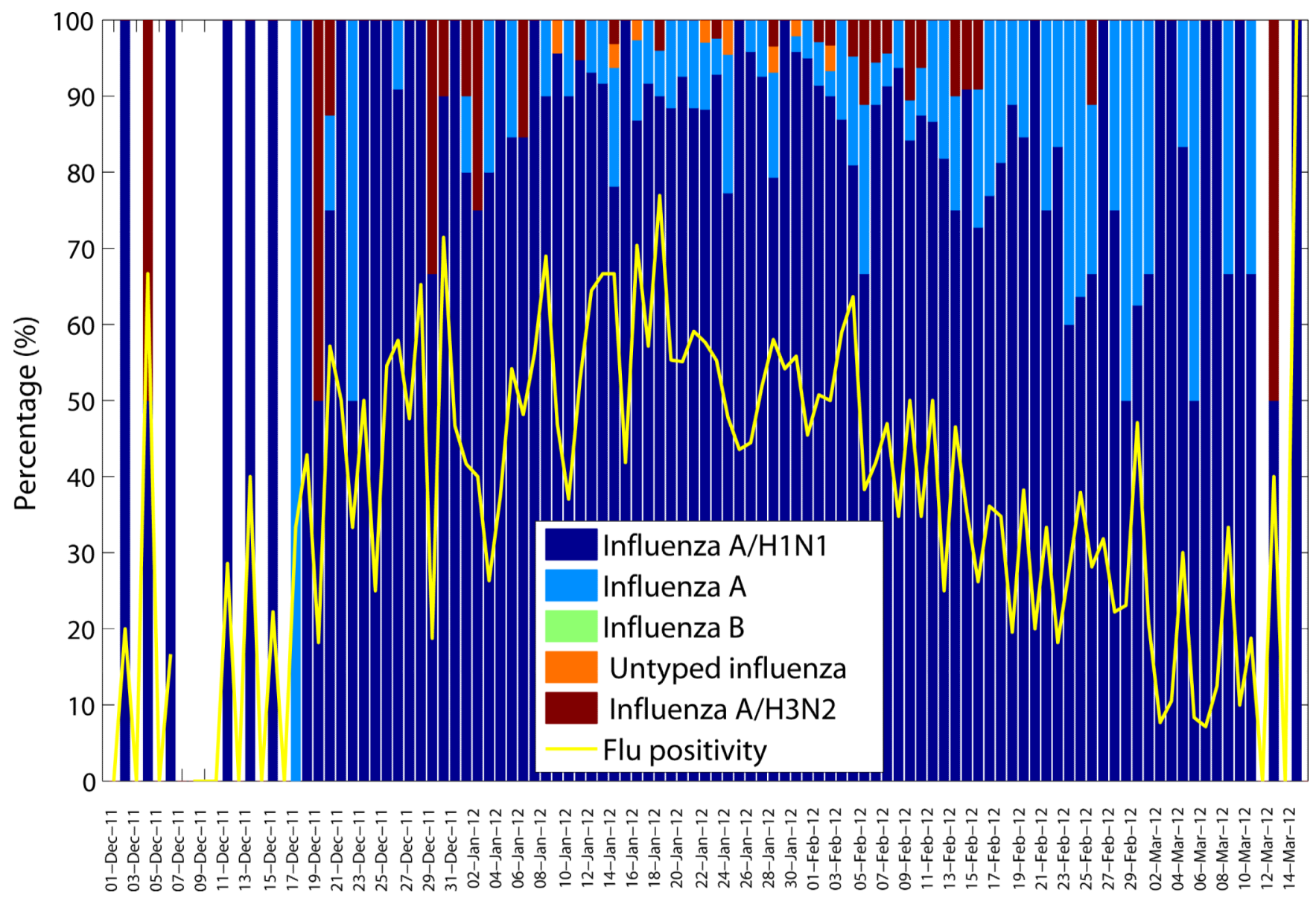

Figure 3.

Daily influenza positivity rates (number of influenza positive SARI hospitalizations/number of tests among SARI hospitalizations) and percentage of influenza specific subtypes among influenza positive tests according to dates of symptom onset (December 1, 2011-March 15, 2012). 

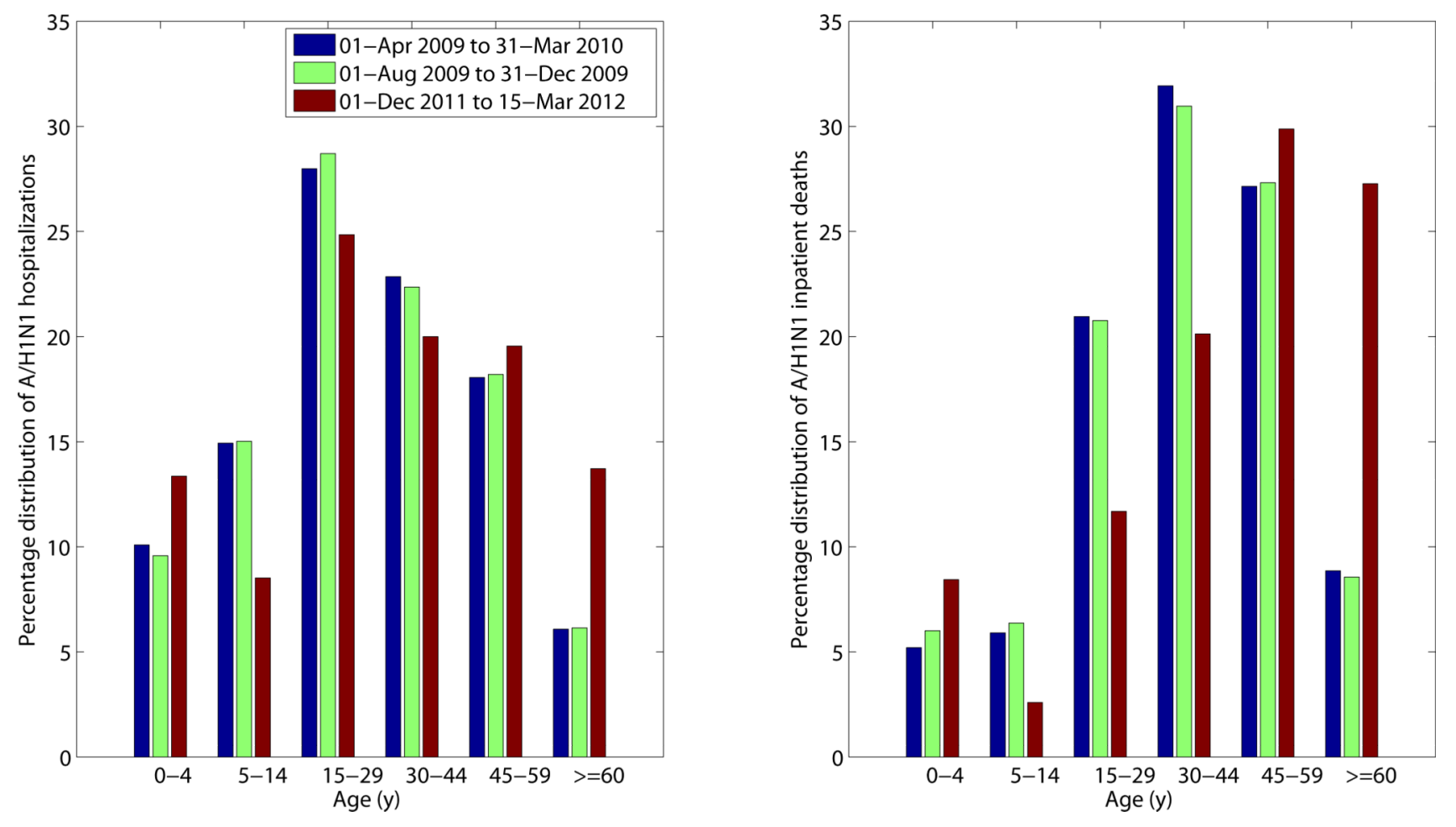

Figure 4.

Age-specific proportions of A/H1N1 influenza hospitalizations (left) and A/H1N1 inpatient deaths (right) for the 2011-2012 winter A/H1N1 influenza epidemic (December 1, 2011March 15, 2012) compared to those of the entire 2009 A/H1N1 pandemic period (April 1, 2009-March 31, 2010) and to the first 70 days of the 2009 fall pandemic wave (August 1, 2019-October 10, 2009). 
Table 1

Characteristics of all SARI hospitalizations and laboratory-confirmed A/H1N1 influenza hospitalizations, Mexico (December 1, 2011 through March 15, 2012) according to dates of symptom onset

\begin{tabular}{lcc}
\hline Variable & SARI hospitalizations & A/H1N1 confirmed hospitalizations \\
\hline Geography & & \\
Central & $3527(46.7)$ & $614(55.1)$ \\
Southern & $1329(17.6)$ & $190(17)$ \\
Other states & $2693(35.7)$ & \\
Demography & & $609(54.6)$ \\
Female & $3860(51)$ & \\
Age (years) & & $149(13.4)$ \\
$0-4$ & $1893(25)$ & $95(8.5)$ \\
$5-14$ & $587(7.8)$ & $277(24.8)$ \\
$15-29$ & $1059(14)$ & $223(20)$ \\
$30-44$ & $1108(14.6)$ & $218(19.6)$ \\
$45-59$ & $1114(14.7)$ & $153(13.7)$ \\
\hdashline 60 & $1808(23.9)$ & $154(13.8)$ \\
Inpatient severity & & \\
Deaths & $443(5.9)$ &
\end{tabular}




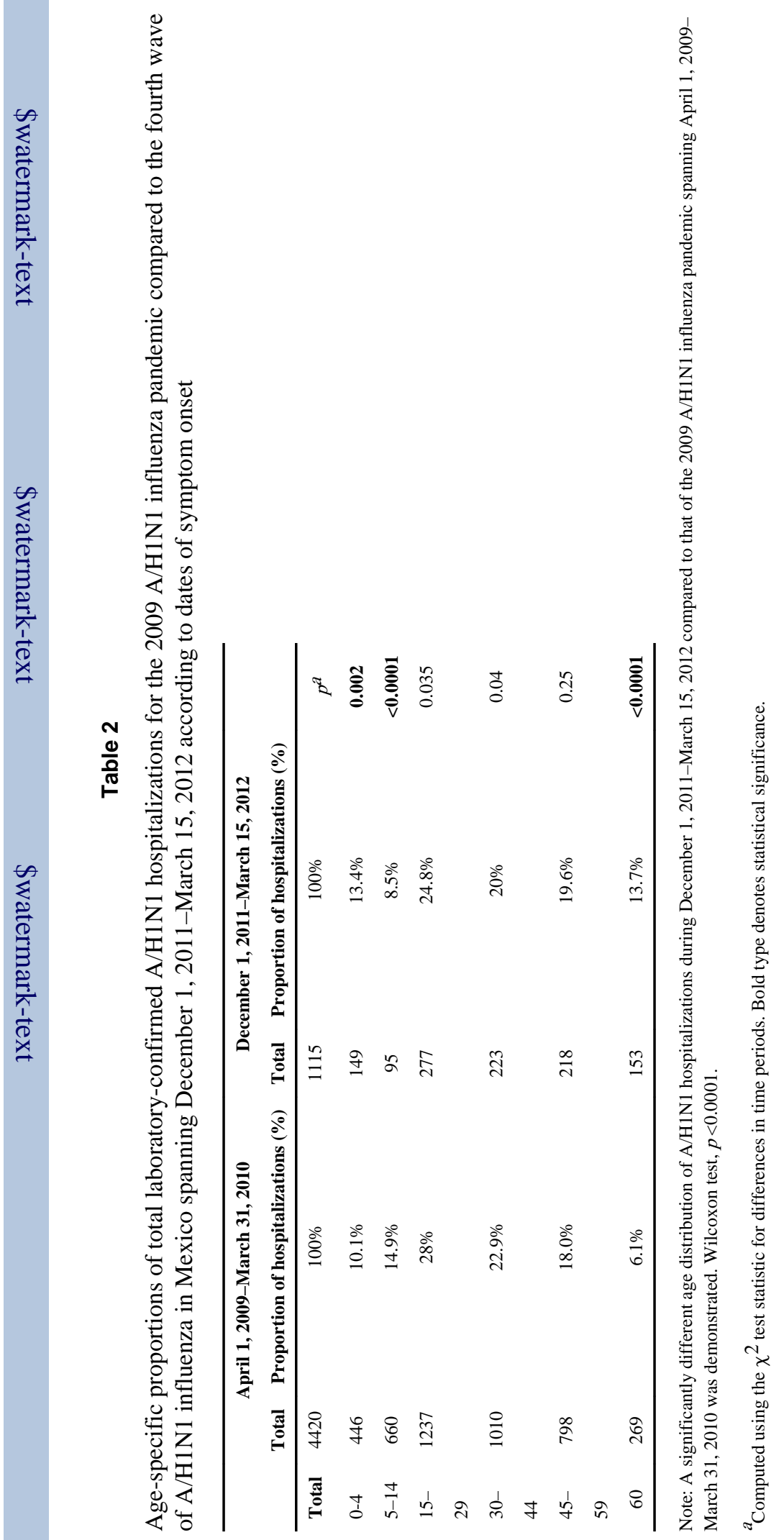

